\title{
The influence of context on intentional service
}

\author{
Salma Najar \\ Centre de Recherche en Informatique \\ Université Paris1 Panthéon-Sorbonne \\ Paris, France \\ Citypassenger, Courtaboeuf, France \\ Salma.Najar@malix.univ-paris1.fr
}

\author{
Manuele Kirsch-Pinheiro \\ Centre de Recherche en Informatique \\ Université Paris1 Panthéon-Sorbonne \\ Paris, France \\ Manuele.Kirsch-Pinheiro \\ (a) univ-paris1.fr
}

\author{
Carine Souveyet \\ Centre de Recherche en Informatique \\ Université Paris1 Panthéon-Sorbonne \\ Paris, France \\ Carine.Souveyet@univ-paris1.fr
}

\begin{abstract}
Several service-oriented approaches promote the intention concept as a way to describe and document services based on user's requirements. However, these approaches have two main limitations: (1) they don't take into account the fact that a user evolves in a context that can influence his intentions, and (2) at the software service level, the corresponding intentional description of these software services is missing. Such a description should be a high level one, which is not directly connected to the software services. The objective of the paper is to propose a semantic service description that considers both intention corresponding to the service and context in which it is supposed to emerge. In addition, the variability embedded in the intentional description can be also affected by the user context. Such influence is also considered in our proposition.
\end{abstract}

Keywords-OWL-S; SOA; Intentional service; context aware service; variability

\section{INTRODUCTION}

Service orientation has been applied for implementing complex business processes in an interoperable and loose coupling way. As part of a business process, services are built in order to satisfy user requirements that can be seen as user intentions pointing out why a user needs a service. In this sense, an intention can be defined as a goal to be achieved by performing a process presented as a sequence of intentions and strategies to the target intention [2].

Although services are supposed to satisfy precise user intentions, their interface focuses the operations service performs. No information concerning user intention behind a service remains on service description. The absence of this information on the service description reinforces the conceptual mismatch [9][22] that exists between end users and service description. Indeed, end users may experiment an increasing difficulty to understand service description, mainly because the nature of such descriptions composed by low-level technical statements. Several works [9][14][19] have considered this conceptual mismatch between software services and user's understanding about business processes implemented by these services. Nevertheless, such works adopt, in their majority, a top-down approach, producing software services from user intentions. Rarely information about user intentions is maintained together with the software service description (e.g. [19][22]). As a result, the search and discovering of software services based on the user intentions they are supposed to satisfy remain difficult.

Besides, the context in which intention emerges remains unexplored. Context information stands for any information that can be used to characterize the situation of an entity (a person, place, or object) considered as relevant to the interaction between a user and an application [6]. Several researches have pointed out the influence of context on business processes [23][24]. Similar to these authors, we believe that business processes are executed in a given context, which can influence such a process. Besides, we believe that user intentions emerge in a given context, which should be observed in order to fully satisfy such an intention. We advocate that an intention is meaningful when considering it in a given context. For us, an intention is not a simple coincidence. It emerges because a user is under a given context. As a consequence, a user will not require a service just because he is located in a given place or under a given context. He does require service because he has an intention that a service can satisfy in this context.

In this paper, we propose a semantic description of services that merges both points of view: user intentions that services are supposed to satisfy, and contexts in which such intentions emerge and that can influence service execution. Such high-level description is built on the top of wellknown semantic Web technology OWL-S [13], by extending it with an intentional and a contextual description for a service. We believe that, by representing both points of view in an integrated semantic descriptor, it will be possible to produce user-centered service search and discovery processes, since such modeling allows combining the use of semantic Web techniques with end user understanding about business process. Semantic description we propose considers the intention corresponding to a service, as well as the context in which the intention is supposed to emerge. It also considers the implication of context information on process variability, by associating contextual conditions to the process variants described at the intentional level.

This paper is organised as follow: Section II presents an overview on related works. Section II introduces the notion of context and its representation. A semantic descriptor for 
intentional and context-aware services is described in section IV. And finally, we conclude in Section V.

\section{RELATED WORKS}

When regarding the literature, one may observe several examples of service-oriented approaches that consider user requirements. Many of these works consider user requirements by considering the user intentions behind service definition. Among these works, it is worth noting works, such as [9][22][19], which propose building software services based on user intentions. These works usually propose specific methodologies (or frameworks) for iteratively building software services, guided by different representations of user intentions. Penserini et al. [19] and Rolland et al. [22] are two examples of such works.

However, in these works, the representation of users intentions is often lost during the building process. As a consequence, the software service description has no concrete information about the intention it is supposed to satisfy. Knowledge about user intention is lost at the software level, representing an important semantic lost (software service is semantically poorer than it would be).

Other works, such as Mirbel et al. [14], prevent loosing such knowledge by using Web semantic technologies. Thus, Mirbel et al. [14] propose search mechanisms that operationalize intention-based services. Nevertheless, these authors do not consider service composition, neither on intentional level, nor on software level.

Besides, none of these works consider the context in which a service is executed or the context in which an intention emerges. Recent works, such as Zhang et al. [29] and Bonino et al.[1], have considered combining both context and user intention on service-orientation. However, the context information, even if it is actually used on such service, is usually limited to the service inputs and outputs. For instance, Bonino et al. [1] consider context information as a way to enrich service input and as a way to discharge the user from filling some input information that can be automatically captured. These authors do not consider the impact of the context information at the intentional level, neither its impact on the service variability.

It is worth noting that, similar to Zhang et al. [29] and Bonino et al. [1], several works have considered enriching service with context information [12][27][26]. Such works have considered using semantic Web technologies for describing context-aware services, which can be defined as services that are able to adapt themselves according to the context in which they are used. Nevertheless, contrary to [2], works such as [26][27] do not consider the user intention, even if they recognize the importance of the final user for the service definition [3].

Inspired by works on context-aware services and on intentional approaches, we propose in this paper a semantic description of context-aware intentional services. Similar to Suraci et al. [26], the proposed description uses semantic Web technologies to describe context information, but it directly associates such a contextual information with the user intention the service is supposed to satisfy. Thus, a complete semantic descriptor that can be explored on both levels is proposed: at a high-level, based on the end user intention the service exposes; and at a low-level, with context that can be potentially used for adaptation purposes.

\section{CONTEXT AND CONTEXT AWARENESS}

The concept of context corresponds to a very wide notion, which is used on a large variety of research domains on computer science. According to Coutaz et al. [5], context entails both recognition and mapping by providing a structured, unified view of the world in which the system operates. This unified view provides a common ground for interpreting an action: "context - the organizational and the cultural context, as much as the physical context, plays a critical role in shaping action, and also in providing people with the means to interpret and understand action" [7].

With the development of new computer devices (tablets, smartphones...), the notion of context has also intensely developed by context-aware computing [6] [15], which is a research field investigating how the user's current context can be exploited for adaptation purposes. Context-awareness can be defined as the capacity of the system to perceive and analyse the user's situation and to adapt itself (i.e., its behaviour, services, interface, etc.) accordingly [6][18].

Works on context-aware computing consider the notion of context as referring to the physical and social situation in which computational devices are embedded [15]. One of the most commonly referenced definitions is proposed by Dey [6], which defines context as: 'any information that can be used to characterize the situation of an entity. An entity is a person, place, or object that is considered relevant to the interaction between a user and an application, including the user and applications themselves'. Dey [6] describes context awareness as a 'property of a system that uses context to provide relevant information and/or services to the user, where relevancy depends on the user's task'.

Context information can stand for a plethora of information, from user's location [4], device resources [20], up to user's agenda and other high level information [10]. It can be applied to select appropriate content [4][10], to adapt content presentation, or even to reconfigure software functionalities [8]. Different categories of context information can then be distinguished. For example, Najar [17] present context according to three categories: (i) environment context representing contextual information about user location, time, social context, etc.; (ii) user context that represents user profile, agenda, Role, activity, etc.; (iii) computational context including contextual information related devices, resource, network, etc.

Nevertheless, in order to be explored for adaptation purposes, context information should be captured and modelled appropriately. Context information is characterized by its high dynamic nature, changing according user mobility and changes on the observed 
environment. The way context information is used depends then on what is observed and how it is represented. In other terms, the context-adaptation capabilities depend on the context model, which is applied [16].

Through the literature, we can observe that various context models and representation approaches, varying from simple key-value structures to ontology-based models. The latter represent an important tendency on most recent works, which is motivated by the capability ontologies gave for enabling knowledge sharing in a non-ambiguous manner and the underlying reasoning possibilities [16]. This tendency follows the evolution of context-aware services, which adhere, in their majority, to a semantic description of such services. In this paper, we also adhere to this tendency, adopting an ontology-based context modelling based on Reichle et al. [20]. This model is coupled with the MUSIC middleware [8], which supplies a context acquisition mechanism as well as a semantic interpretation for observed context information.

\section{CONTEXT Aware InTENTIONAL SERVICE DESCRIPTION}

In this paper, we consider that services are executed in a given context, but also that services are supposed to satisfy certain user intentions. However, as pointed out in section II, currently service description does not necessarily represents both aspects. Thus, we propose to bring services one level up, by proposing a semantic description of services that merges both user intentions that services are supposed to satisfy, and context in which such intentions emerge and that can influence service execution.

In order to propose such a semantic description, a rich description language is needed. According to Suraci et al. [26], the latest research in Service Oriented Computing (SOC) recommends the use of the OWL-S for semantically describe services. Even if OWL-S is tailored for Web services, it is rich enough to describe any service [26].

OWL-S [13] describes services in three interrelated subontologies. The first part concerns the service profile that expresses what the service does. The second one represents the process model that represents the service's behaviour as a process and describes how it works. The third part refers to the grounding that maps the constructs of the process model onto detailed specification of message, formats and protocols. The first part, service profile, exposes the service interface for search and discovery purposes. The second one, process model, describes service composition, while the third part, service grounding, indicates how to call the service.

Thus, we propose to extend OWL-S service description with an intentional and a contextual description for services, in order to produce real user-centered service search and discovery processes. Such extension affects essentially the service profile and the process model behind the service. Service profile is affected since the interface of the service is raised up to an intentional level. A service is not anymore just a set of operations. A service should also correspond to an intention that emerges in a given context. Process model is affected by the same reason: process model does not correspond only to a software level process; it might correspond to an intentional composition, i.e. a composition of several low-level intentions on a higher level one. Such intentional composition can point out possible variants on process execution. Such variants can be associated with a given context in which a variant is better suited than another one. We associate contextual conditions to the process variants described at the intentional level.

\section{A. Intention Description}

Several researches in service engineering [19][21][28] focus on the adoption of intention-based approaches from requirements engineering domain to identify user intentions. This vision is the base for several intention-based approaches [9][1][25], which propose to take into account user intentions, when proposing business services.

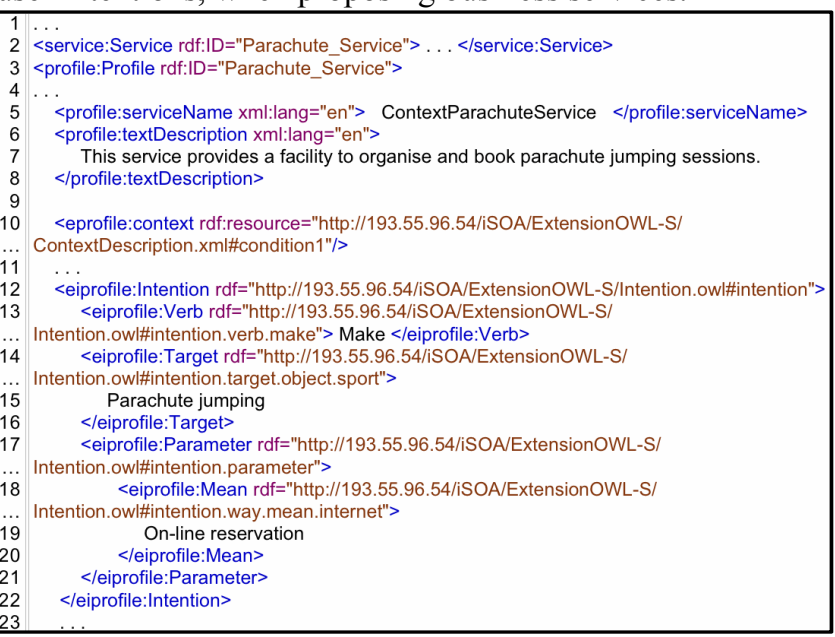

Figure 1: Intentional description of a service

According to an intentional perspective, a user has a particular intention when requesting a service. The service is proposed in order to satisfy this intention. Hence, it emerges a user centric vision on the service orientation that takes into account the user intention. An intention becomes central on the service definition. We propose to enrich OWL-S service description with the intention associated to it. We extend OWL-S profile, including on it the intention that a service can satisfy. This is done by a new parameter named "intention", specialising the service profile parameters. It is formulated according to a specific template, as in [22], in which an intention is represented by a verb, a target and a set of parameters. Figure 1 illustrates this OWL-S service profile extension. A service is associated with the intention "make parachute jumping", which is described according the template "verb target parameters", using the extended OWL-S elements.

By extending OWL-S service profile with an intentional description, we allow service providers to expose the principal intention the service is designed to satisfy. 
Furthermore, this main intention can actually be seen as a high level intention that can be decomposed into lower level intentions. Such an intentional composition has been proposed by authors in [9][22], as a way to introduce variability on business process realisation. This variability is justified by the need to introduce flexibility and adaptability in intention achievement [22].

While the OWL-S process model describes the technical composition of a service, representing software components that are combined to supply the required service, nothing is proposed in OWL-S for describing the intentional composition. Intentional composition represents a way to expose not only lower level intentions necessary to satisfy service intention, but also the different possibilities for satisfying this intention. The technical composition supplies the technical elements necessary for service execution, while intentional composition provides an understanding of the service and of the diverse forms of satisfying service intention, from final user's point of view.

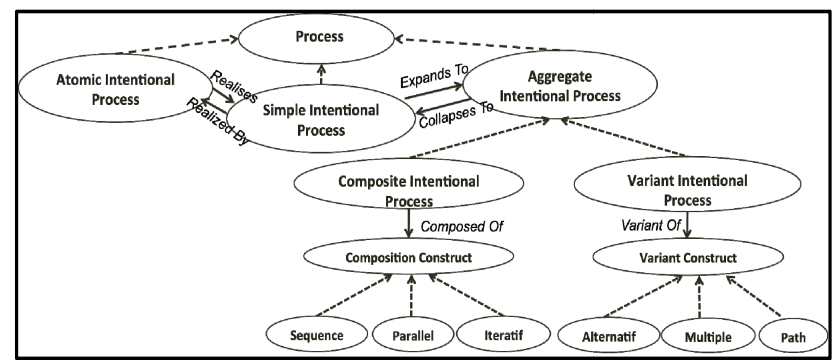

Figure 2: Composing intentions in OWL-S process model

Thus, we propose to extend OWL-S process model by including the specification of an intentional service process, as presented in Figure 2. Such extension takes the form of a new element on OWL-S process model, named aggregate Intentional Process, which encompass two kinds of intentional process, a composite one and a variant one.

From the one side, the composite intentional process reflects the precedence/succession relationship between their intentions. Such relationships are specified using composition constructs such as Sequence, Parallel and Iterative. From the other side, the variability is represented by the variant intentional process, which uses constructs such as multiple, alternative and path.

This separation, based on [9][21], intends to reinforce variability aspects on intentional achievement.

\section{B. Context Description}

As pointed out in section III, context information forms a common ground for interpreting and understanding an action. More than just actions, we advocate that context information contributes to fully understanding user intentions. We believe that user intentions emerge in a given context, in which they are meaningful. Conversely, context description is only meaningful when associated with an intention. Thus, we propose to enrich the OWL-S service profile description with context information that characterizes an intentional service. From the one side, we describe the context referring to the conditions under which it is most appropriate to call this service. This represents the context in which the intention emerges, in which service is supposed to be fully useful for the user. From the other side, we describe the context in which a service can be executed. It refers to the context conditions under which a service is running on the service supplier.

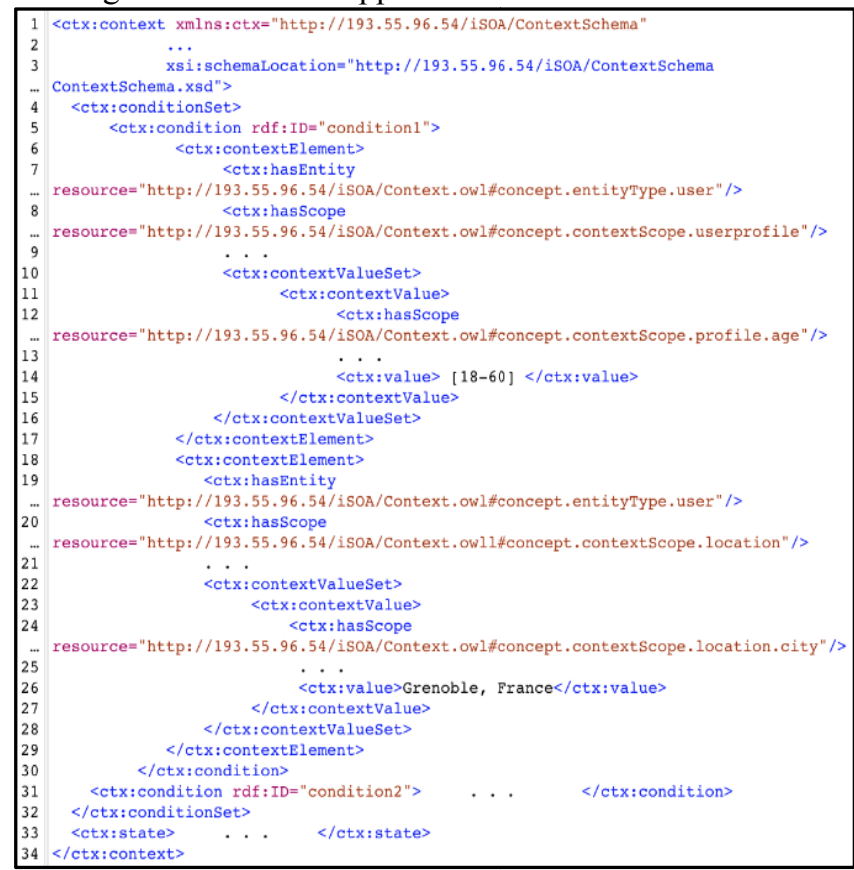

Figure 3: Context description file referred by a service

Both descriptions are kept in an external file, illustrated by Figure 3 by the service profile. Based on Kirsch et al. [11], we enrich OWL-S service profile with a context attribute, which represents a URL pointing to this context description file. Such separation is needed since context information is dynamic and cannot be statically stored on the service profile description. Context properties related to service execution can evolve (for instance, server load may affect properties of services running on it), whereas service description is supposed to be static. It is not supposed to change in short intervals of time (as context information does) [11]. Thus, the description of context information on an external file allows service provider to easily update such context information related to the service description itself. Figure 3 illustrates such context description file, referred by the service represented in Figure 1 (line 10). By referring this context description in the service profile, the service provider exposes that the intention "make parachute jumping", associated with this service, emerges in the context described by the file shown in Figure 3, acting as a contextual pre-condition for executing this service. Thus, the service provider indicates that the service in Figure 1 is adapted for adult users (lines 6-17) and for location near to Grenoble, France (18-29). 


\section{Resource Description}

A service with an intentional description can be seen as an intentional service, as defined by [9][22] that has, in addition, a semantic and a context-aware description. As an intentional service, it can be an aggregation (composite or variant) of low-level intentions (as mentioned in section IV.A). Each intentional service acts as a fragment of process implemented by the software service, handling input information in order to satisfy its corresponding intention and resulting in some output information. Input and output of an intentional service describes, respectively, an initial and a final situation, expressed as set of states over resources handled by the service. Such initial and final situations are crucial for intentional composition, since they are supposed to guide the satisfaction of high-level intention associated with the aggregate service.

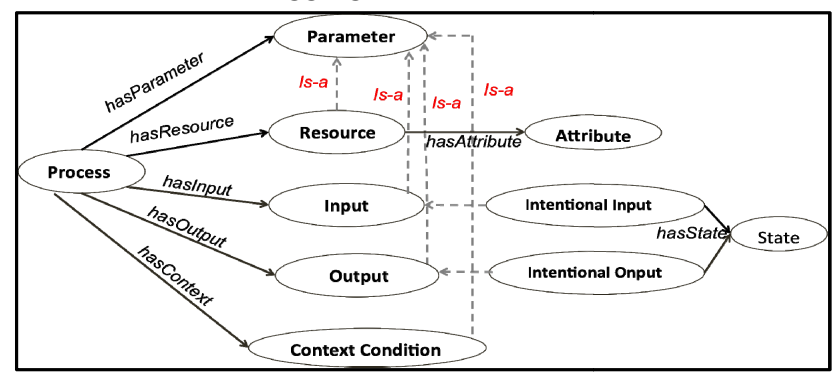

Figure 4: Parameter description in OWL-S process model

In order to express initial and final situations necessary to intentional composition, we introduced the notion of resource on OWL-S, as presented in Figure 4 . A resource represents a class of objects, with its corresponding attributes, that are manipulated by an intentional service. For instance, a service implementing the intention "book parachute jumping" will manipulate a "booking" resource, with a "confirmation" attribute. Then, when the resource is used as intentional input or output parameter, a state can be assigned to the resource. The element state allows then attaching values to each resource attribute. Figure 5 illustrates the resource "booking" used as intentional input with a state defined by the attribute confirmation with the value "pending".

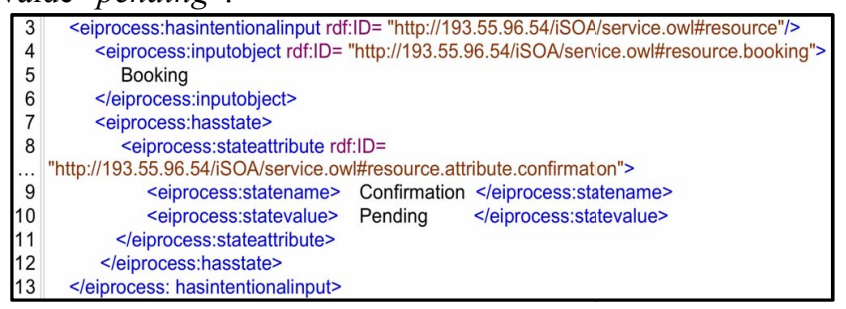

Figure 5: Intentional input in OWL-S process model

In addition to traditional resource manipulated by an intentional service, we believe that variability on intention achievement may depend on external factors, including context information. Each variant may then have some context condition in which it is most appropriate to use it. In order to consider context influence on intentional variants, we propose including context information on the process variability, by associating contextual conditions to each process variant described at the intentional level (see Figure 3 ). Our purpose is to permit the selection of the most suited variant for satisfying the user intention in his current context. This context condition grants a more dynamic variability to intentional process by adding contextual information that will guide the choice of the variant according to the context condition.

Thus, we extend OWL-S process model by including on it an intentional composition (explained in section IV.A), in which each variant may indicate a contextual condition through the element contextCondition. Similar to the context element on service profile (see section IV.B), this element points out to an external file containing context description. Actually, for representing these contextual conditions, we adopt the same approach, based on [11], than for describing overall service context. However, different from [11], our context file now integrates several context descriptions, representing not only context directly associated with main service intention (as explained in section IV.B), but also contextual conditions associated with particular variants. Thus, service provider can fully expose all possible variants considered by implemented business process. Figure 6 illustrates this OWL-S process model extension through a contextual condition pointing out the context description file presented in Figure 3.

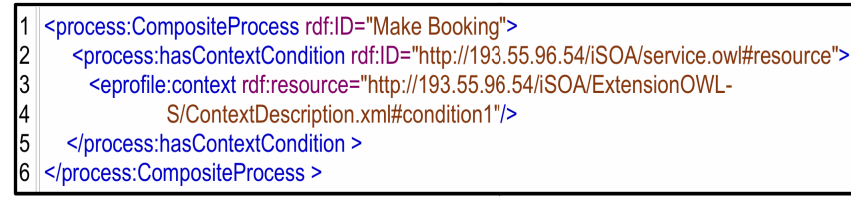

Figure 6: Context condition in OWL-S process model

\section{CONCLUSION}

In this paper, we propose a semantic description of services representing both user intentions that services are supposed to satisfy and contexts in which such intentions emerge and which influence service execution. We consider these approaches as complementary. We advocate that an intention is only meaningful when considering the context in which it emerges, and conversely, the context description is only meaningful when associated with a user intention.

From the one side, we propose to enrich OWL-S service description by extending it with an intentional and a contextual description for a service. From the other side, we emphasize the fact that context can influence variability process, by making explicit this influence on intentional process model, and mainly on process variability.

By representing both points of view in an integrated semantic descriptor, we enable the expression of services that can adapt themselves to context of use and that represent a formulated user requirement. By considering the use of semantic Web technologies with end user 
understanding about process, we expect to progress towards a real user-centred service discovery processes.

In order to progress on this sense, our next step is to propose a service discovery mechanism that can take into account the improvements we propose here. Our intention is to propose a mechanism to discover and to select the most appropriate service according to the user requirements expressed in a given context.

\section{REFERENCES}

[1] Bonino da Silva Santos L.O., Pires L.F., Van Sinderen M.: A Goal-Based Framework for Dynamic Service Discovery and Composition, Int Workshop on Architectures, Concepts and Technologies for Service Oriented Computing, Porto, Portugal, 2008, 67-78.

[2] Bonino da Silva Santos L.O., Guizzardi G., Pires L.F., Van Sinderen M., From User Goals to Service Discovery and Composition, ER Workshops 2009, 265-274

[3] Brnsted J., Hansen K., Ingstrup M., Service Composition Issues in Pervasive Computing, Pervasive Computing, IEEE, vol. $9 \mathrm{n}^{\circ} 1,2010,62-70$

[4] Burrell J., Gray G., Kubo K., Farina N., Context-aware computing: a test case, Holmquist, L. \& Borriello, G. (Eds.), 4th Conf on Ubiquitous Computing, LNCS 2498, Springer, 2002, 1-15

[5] Coutaz, J.; Crowley, J.; Dobson, S., Garlan, D., Context is the key, Communication of the ACM, vol. $48 \mathrm{n}^{\circ} 3,2005,49-53$

[6] Dey A., Understanding and using context, Personal and Ubiquitous Computing, Vol. $5 \mathrm{n}^{\circ} 1,2001,4-7$

[7] Dourish, P., Seeking a foundation for context-aware computing, Human Computer Interaction, vol. $13 \mathrm{n}^{\circ} 2-4$, 2001, 229-241

[8] IST-MUSIC, December, 2010 from http://www.ist-music.eu/

[9] Kaabi R.S., Souveyet C., Capturing intentional services with business process maps, RCIS, 2007, 309-318.

[10] Kirsch-Pinheiro M., Gensel J., Martin H., Representing Context for an Adaptative Awareness Mechanism, G.-J. de Vreede; L.A. Guerrero, G.M.Raventos (Eds.), LNCS 3198 - X Workshop on Groupware (CRIWG 2004), Springer, 339-348.

[11] Kirsch-Pinheiro M., Vanrompay Y., Berbers Y., Contextaware service selection using graph matching, 2nd Non Functional Properties and Service Level Agreements in Service Oriented Computing Workshop (NFPSLA-SOC'08), ECOWS 2008. CEUR Workshop proceedings, Vol. 411

[12] Maamar Z., Benslimane D., Narendra N.C., What can context do for web services?, Communication of the ACM, Vol. $49 \mathrm{n}^{\circ}$ 12, 2006, 98-103

[13] Martin D., Paolucci M., Mcilraith S., Burstein M., Mcdermott D., Mcguinness D., Parsia B., Payne T., Sabou M., Solanki M., Srinivasan N., Sycara K., Bringing Semantics to Web Services: The OWL-S Approach, Cardoso, J. \& Sheth, A. (Eds.), SWSWPC 2004, LNCS 3387, Springer, 2004, 26-42

[14] Mirbel I, Crescenzo P., From end-user's requirements to Web services retrieval: a semantic and intention-driven approach, J.-H. Morin, J. Ralyte, M. Snene, "Exploring service science",
First International Conference, IESS 2010, LNBIP 53, Springer, 2010, 30-44.

[15] Moran, T., Dourish, P., Introduction to this special issue on context-aware computing, Human-Computer Interaction, vol.16 n²-3, 2001, 87-95

[16] Najar S., Saidani O., Kirsch-Pinheiro M., Souveyet C., Nurcan S., Semantic representation of context models: a framework for analyzing and understanding. J. M. GomezPerez, P. Haase, M. Tilly, and P. Warren (Eds), $1^{\text {st }}$ Workshop on Context, information and ontologies (CIAO 09), European Semantic Web Conference (ESWC'2009), ACM, 1-10

[17] Najar S.: Context-Aware Intentional Service Framework for

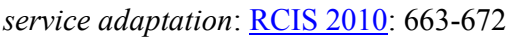

[18] O'Hare G., O'Grady M., Addressing mobile HCI needs through agents: Paternò, F. (ed.), Mobile HCI 2002, LNCS 2411. Springer, (2002), 311-314.

[19] Penserini L., Perini A., Susi A., High Variability Design for Software Agents: Extending Tropos. ACM Transaction on autonomous and Adaptative Systems, Vol. 2 n4 $^{\circ} 2007$

[20] Reichle R., Wagner M., Khan M., Geihs K., Lorenzo L., Valla M., Fra C., Paspallis N., Papadopoulos G.A., A Comprehensive Context Modeling Framework for Pervasive Computing Systems. In 8th IFIP Conf on Distributed Applications and Interoperable Systems. Springer, 2008.

[21] Rolland C., Kraiem N., Kaabi R.S, On ISOA : Intentional Service Architecture, $19^{\text {th }}$ Conf on Advanced Information Systems Engineering, CAISE'07,Trondheim, Norway, Springer, 2007, 158-172.

[22] Rolland C., Kirsch-Pinheiro M., Souveyet C., An Intentional Approach to Service Engineering, IEEE Transactions on Service Computing, Vol.3 n4, 2010, 292-305.

[23] Rosemann M., Recker J., Flender C., Contextualization of Business Processes. Int. J. Business Process Integration and Management, Vol. 1, n¹/2/3, 2007

[24] Saidani O., Nurcan S., Towards Context Aware Business Process Modeling, 8th Workshop on Business Process Modeling, Development, and Support (BPMDS'07), CAiSE'07

[25] Stollberg M., Norton B., A Refined Goal Model for Semantic Web Services. ICIW 2007: Vol.17

[26] Suraci V., Mignanti S., Aiuto A., Context-aware Semantic Service Discovery, 16th IST Mobile and Wireless Communications Summit, 2007, 1-5

[27] Taylor N., Robertson P., Farshchian B., Doolin K., Roussaki I., Marshall L., Mullins R., Druesedow S., Dolinar K., Pervasive Computing in Daidalos, Pervasive Computing, vol. $10, \mathrm{n}^{\circ} 1,2011,74-81$

[28] Van Lamsweerde A., Requirements Engineering in the year 2000: A research perspective. 22nd Int Conf on Software Engineering, Limerick, Ireland, 2000.

[29] Zhang K., Li Q., Sui Q., A Goal-driven Approach of Service Composition for Pervasive Computing : 1st Int Symposium on Pervasive Computing and Applications, August, 2006, 593598. 